\title{
Accuracy of the ELITe MGB assays for the detection of carbapenemases, CTX-M, Staphylococcus aureus and mecA/C genes directly from respiratory samples
}

\author{
M. Boattini ${ }^{a, *}$, G. Bianco ${ }^{a}$, M. Iannaccone ${ }^{a}$, L. Charrier $^{b}$, A. Almeida ${ }^{c, d}$, \\ G. De Intinis ${ }^{a}$, R. Cavallo ${ }^{a}$, C. Costa ${ }^{a}$ \\ a Microbiology and Virology Unit, University Hospital Città della Salute e della Scienza di Torino, Turin, Italy \\ ${ }^{\mathrm{b}}$ Department of Public Health and Paediatrics, University of Torino, Turin, Italy \\ ' Department of Internal Medicine 4, Hospital de Santa Marta, Central Lisbon Hospital Centre, Lisbon, Portugal \\ ${ }^{\mathrm{d}}$ NOVA Medical School, Universidade Nova de Lisboa, Lisbon, Portugal
}

\section{A R T I C L E I N F O}

\section{Article history:}

Received 24 October 2019

Accepted 30 December 2019

Available online 10 January

2020

\section{Keywords:}

Antibiotic resistance genes Molecular assay

Bronchoalveolar lavage fluid Antimicrobial stewardship

Respiratory samples

Pneumonia

\section{$S U M M A R Y$}

Introduction: Bacterial lower respiratory tract infections (BLRTI) may represent serious clinical conditions which can lead to respiratory failure, intensive care unit admission and high hospital costs. The detection of carbapenemase- and extended-spectrum $\beta$-lactamase (ESBL)-producing Enterobacterales, as well as meticillin-resistant Staphylococcus aureus (MRSA), has become a major issue, especially in healthcare-associated infections. This study aimed to determine whether molecular assays could detect genes encoding carbapenemases, ESBL and MRSA directly from respiratory samples in order to expedite appropriate therapy and infection control for patients with BLRTI.

Methods: The carbapenem-resistant enterobacterales (CRE), ESBL and MRSA/SA ELITe MGB assays were performed directly on 354 respiratory specimens sampled from 318 patients admitted with BLRTI. Molecular results were compared with routine culturebased diagnostics results.

Results: Positive (PPV) and negative (NPV) predictive values of the CRE ELITe MGB kit were 75.9\% [95\% confidence interval $(\mathrm{Cl}) 60.3-86.7]$ and $100 \%$, respectively. PPV and NPV of the ESBL ELITe MGB kit were $80.8 \%(95 \% \mathrm{Cl} 63.6-91.0)$ and $99.1 \%$ (95\% Cl 96.6-99.8), respectively. PPV and NPV of the MRSA/SA ELITe MGB kit were $91.7 \%(95 \% \mathrm{Cl} 73.7-97.7)$ / $100 \%$ and $98.3 \%$ (95\% Cl 89.8-99.3)/96.8\% (95\% Cl 81.6-99.5), respectively.

Discussion: Validity assessment of molecular assays detecting the main antibiotic resistance genes directly from respiratory samples showed high accuracy compared with culture-based results. Molecular assays detecting the main carbapenemase, ESBL, S. aureus and meticillin resistance encoding genes provide an interesting tool with potential to expedite optimization of antibiotic therapy and infection control practices in patients with BLRTI.

( 2020 The Healthcare Infection Society. Published by Elsevier Ltd. All rights reserved.

\footnotetext{
* Corresponding author. Address: Microbiology and Virology Unit, University Hospital Città della Salute e della Scienza di Torino, Corso Bramante 88/90, 10126, Turin, Italy. Tel.: +390116335948.

E-mail address: matteo.boattini@unito.it (M. Boattini).
} 


\section{Introduction}

Bacterial lower respiratory tract infections (BLRTI), including bronchitis, pneumonia and infectious exacerbations in chronic lung disease, can represent serious clinical conditions which can lead to respiratory failure, intensive care unit admission, prolonged admission and high hospital costs [1-7]. Patients admitted with BLRTI are frequently prescribed broadspectrum empirical antibiotics; timely identification of pathogens is necessary to support antibiotic stewardship and therefore reduce the risk of selection of antibiotic resistance. Detection of carbapenemase- and extended-spectrum $\beta$-lactamase (ESBL)-producing Enterobacterales and meticillinresistant Staphylococcus aureus (MRSA) has important implications for both antimicrobial therapy and infection control, especially in healthcare-associated infections.

A positive microbiological diagnosis in BLRTI may only be made in approximately $30 \%$ of cases [8] and, as commensal and colonizing micro-organisms complicate the analysis, conventional phenotypic diagnostics for respiratory samples typically takes approximately $48-72 \mathrm{~h}$, hampering antimicrobial stewardship. Molecular tests for genes encoding carbapenemases, ESBLs and MRSA have been successfully applied directly to blood culture samples $[9,10]$ but there is limited published evidence about their performance on respiratory samples [11-15].

The ELITe InGenius (ELITechGroup Molecular Diagnostics, Turin, Italy) platform is an integrated system that automatically performs nucleic acid extraction, real-time polymerase chain reaction (PCR) and interpretation of results in less than $3 \mathrm{~h}$. The carbapenem-resistant enterobacterales (CRE) and ESBL ELITe MGB kits are qualitative multiplex real-time PCR assays for the detection of the most prevalent carbapenemase- and ESBLencoding genes in Enterobacterales. The CRE ELITe MGB kit

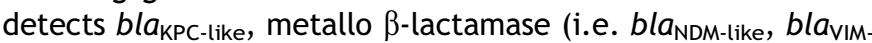
like, $b$ ( $\left.a_{\text {IMP-like }}\right)$ and bla $a_{\text {OXA-48-like }}$ genes, and the ESBL ELITe MGB kit detects $b$ la $a_{\mathrm{CTX}-\mathrm{Ms}}$ genes belonging to groups 1 (including CTX$M-15)$ and 9 (including CTX-M-14). The MRSA/SA ELITe MGB kit is a multiplex assay that simultaneously detects a conserved sequence of the $S$. aureus, mecA gene and its homologue mecA $\mathrm{LGA251}_{\text {(mecC). }}$

The aim of this study was to evaluate the performance of CRE, ESBL and MRSA/SA ELITe MGB assays directly on respiratory samples, including comparing real-time PCR cycle threshold $(\mathrm{Ct})$ values with bacterial load quantification.

\section{Methods}

\section{Routine culture-based microbiological diagnostics}

At the Microbiology and Virology Unit of Azienda OspedalieroUniversitaria Città della Salute e della Scienza di Torino in Turin, Italy, respiratory samples were subjected to Gram staining and culture on appropriate solid medium at the time of arrival at the laboratory. Matrix-assisted laser desorption/ionization-time of flight mass spectrometry analysis was used for bacterial identification, and antimicrobial susceptibility testing was carried out on overnight subcultures using Microscan WalkAway plus system (Beckman Coulter, Brea, CA, USA) according to the manufacturer's instructions. Antimicrobial susceptibilities were interpreted according to EUCAST breakpoints as updated in 2019 [16]. The Total ESBL Confirm kit (Rosco, Taastrup, Denmark) was used to identify ESBL production if cefotaxime and/or ceftazidime minimal inhibitory concentrations (MICs) were $>1 \mathrm{mg} / \mathrm{L}$. The Mastdiscs combi Carba plus disc system (Mast Group Ltd, Bootle, UK) was used to assess carbapenemase producers when meropenem MIC was $>0.125 \mathrm{mg} / \mathrm{L}$. Detection of carbapenem resistance genes was performed using the Xpert Carba-R assay (Cepheid, Sunnyvale, CA, USA).

\section{Specimen collection and study design}

Respiratory samples included in the study were those submitted for standard of care bacterial culture from January to June 2019. They were selected at random based on sample type, integrity and amount of remnant specimen. Lower respiratory tract specimens included: sputum, tracheal aspirate (TA), bronchoaspirate (BA) and bronchoalveolar lavage (BAL). The administration of antibiotics before specimen collection was not assessed.

The ELITe MGB assays were performed directly on 354 respiratory specimens sampled from 318 patients. The CRE and ESBL ELITe MGB kits were assayed on sputum $(N=7)$, TA $(N=16)$, BA $(N=16)$ and BAL $(N=202)$, and the MRSA/SA ELITe MGB kit was tested on sputum $(N=35)$, TA $(N=25)$, BA $(N=15)$ and BAL $(N=38)$. Two-hundred microlitres of a 1:4 dilution in dithiothreitol solution (Sputasol, Oxoid Ltd, Basingstoke, UK), previously heated in a thermoblock at $90^{\circ} \mathrm{C}$ for $5 \mathrm{~min}$, was used for sputum, TA and BA, whereas $200 \mu \mathrm{L}$ of a 1:2 dilution in dithiothreitol solution was used for BAL. The ELITe MGB kits' internal control and positive and negative controls were used as described previously [10]. The total ELITe MGB assay test run time is $2 \mathrm{~h} 12 \mathrm{~min}$ with data analysis available immediately after the run. The total cost of the ELITe MGB assays includes reagents (approximately $£ 25$ per sample including DNA extraction), staff time and platform ELITe InGenius rental.

Molecular results were compared with routine culture-based microbiological diagnostics results to estimate the accuracy of genotypic analysis. Molecular results for the CRE, ESBL and MRSA/SA targets were interpreted as shown in Table I. All cycles with a $\mathrm{Ct}$ value $>35$ were considered negative for detectable signal. Presence of $S$. aureus and mecA/C targets at the same relative quantity ( $\Delta C$ t between the two targets $<2$ ) was considered indicative of MRSA (to mitigate against the potential for detection of meticillin-susceptible $S$. aureus together with meticillin-resistant coagulase-negative staphylococci).

$\mathrm{Ct}$ values were also compared with the quantitative culture results in order to maximize the potential clinical impact of the molecular results.

This study was conducted in accordance with the Declaration of Helsinki. Formal ethical approval was not required by the study centre's institutional review board as the samples were anonymized and de-identified before being obtained by the study team.

\section{Statistical analysis}

Accuracy, sensitivity, specificity and positive (PPV) and negative (NPV) predictive values of the CRE, ESBL and MRSA/SA 
Table I

Interpretation of molecular results on respiratory samples for the detection of carbapenemase, extended-spectrum $\beta$-lactamase (ESBL), Staphylococcus aureus and meticillin resistance encoding genes

\begin{tabular}{lcll}
\hline CRE ELITe MGB kit & ESBL ELITe MGB kit & \multicolumn{1}{c}{ Interpretation } & \multicolumn{1}{c}{ Report } \\
\hline+ & - & Carbapenemase encoding gene & KPC, NDM-IMP-VIM, OXA-48 DNA detected \\
+ & + & Carbapenemase and ESBL & Both KPC, NDM-IMP-VIM, OXA-48 \\
& & encoding genes & DNA detected and CTX-Ms DNA detected \\
- & + & ESBL encoding gene & CTX-M DNA detected \\
- & - & Neither carbapenemase & Neither KPC, NDM-IMP-VIM, OXA-48 \\
& & nor ESBL encoding genes & nor CTX-Ms DNA detected \\
\hline MRSA/SA ELITe MGB kit & & & \\
\hline S. aureus & mecA/C & & \\
\hline+ & + & $\Delta C t<2$, MRSA & MRSA DNA detected \\
+ & + & $\Delta C t>2$, MSSA & MSSA DNA detected \\
+ & - & MSSA & MSSA DNA detected \\
- & + & No S. aureus & No S. aureus DNA detected \\
- & - & No S. aureus & No S. aureus DNA detected \\
\hline
\end{tabular}

CRE, carbapenem-resistant enterobacterales; MSSA, meticillin-susceptible S. aureus; MRSA, meticillin-resistant S. aureus; Ct, cycle threshold. All cycles with a $\mathrm{Ct}$ value $>35$ were considered negative.

ELITe MGB kits with $95 \%$ confidence intervals $(95 \% \mathrm{Cl})$ were computed.

The Shapiro-Wilk test was performed to verify the normality of distribution of quantitative variables. Analysis of variance with Bonferroni's correction was carried out to assess whether significant differences in $\mathrm{Ct}$ values could be detected between quantitative culture groups [negative, 1000-10,000 colonyforming units $(\mathrm{cfu}) / \mathrm{mL}$ and $>25,000 \mathrm{cfu} / \mathrm{mL}$ for carbapenemase- and ESBL-producing Enterobacterales; negative, $1000-10,000 \mathrm{cfu} / \mathrm{mL}, 10,000-50,000 \mathrm{cfu} / \mathrm{mL}$ and $>50,000 \mathrm{cfu} /$ $\mathrm{mL}$ for $\mathrm{S}$. aureus). $P$-values $<5 \%$ were considered significant. All analyses were performed using Stata 14 (Stata Corp., College Station, TX, USA).

\section{Results}

\section{Detection of carbapenemase, CTX-M and S. aureus and mecA/C genes}

Table II shows the comparison between molecular and conventional phenotypic results. Among the 241 clinical specimens, bla $a_{K P C \text {-like }}$ was detected in $29(12 \%)$ specimens by the CRE ELITe MGB kit. Twenty-two (75.9\%) of these samples were confirmed by culture. Five of the seven false-positive samples were from patients who became culture-positive for Klebsiella pneumoniae carbapenemase-producing K. pneumoniae (TA $N=2$; $B A N=1$; urine culture $N=1$; rectal swab $N=1$ ) in the subsequent 7 days. bla $a_{\mathrm{CTX}-\mathrm{M} \text {-like }}$ was detected in $26(10.8 \%)$ specimens by the ESBL ELITe MGB kit, of which 21 $(80.8 \%)$ were confirmed by culture. Two of the five false-

Table II

Performance of the carbapenem-resistant enterobacterales (CRE), extended-spectrum $\beta$-lactamase (ESBL) and meticillin-resistant Staphylococcus aureus (MRSA)/S. aureus (SA) ELITe MGB kits on respiratory samples compared with conventional phenotypic results

\begin{tabular}{|c|c|c|c|c|c|c|c|c|c|}
\hline \multicolumn{2}{|c|}{ CRE and ESBL ELITe MGB kits } & \multicolumn{3}{|c|}{$\begin{array}{c}\text { Conventional phenotypic results } \\
\text { Respiratory samples } \\
N=241\end{array}$} & Accuracy & $\begin{array}{l}\text { Sensitivity } \\
(95 \% \mathrm{Cl})\end{array}$ & $\begin{array}{l}\text { Specificity } \\
(95 \% \mathrm{Cl})\end{array}$ & $\begin{array}{c}\text { PPV } \\
(95 \% \mathrm{Cl})\end{array}$ & $\begin{array}{c}\text { NPV } \\
(95 \% \mathrm{Cl})\end{array}$ \\
\hline \multirow[t]{2}{*}{ bla $a_{\mathrm{KPC}-\text { like }}$} & Positive & 22 & & 7 & \multirow[t]{2}{*}{$97.1 \%$} & \multirow{2}{*}{$\begin{array}{l}100 \% \\
(85.1-100)\end{array}$} & $96.8 \%$ & $75.9 \%$ & $100 \%$ \\
\hline & Negative & 0 & & 212 & & & $(93.6-98.4)$ & $(60.3-86.7)$ & \\
\hline bla $a_{\text {CTX-M-like }}$ & Positive & 21 & & $\begin{array}{r}5 \\
213\end{array}$ & $97.1 \%$ & $\begin{array}{l}91.3 \% \\
(73.2-97.6)\end{array}$ & $97.7 \%$ & $80.8 \%$ & $99.1 \%$ \\
\hline \multirow{2}{*}{\multicolumn{2}{|c|}{ MRSA/SA ELITe MGB kit }} & \multicolumn{2}{|c|}{$\begin{array}{l}\text { Respiratory samples } \\
\qquad N=113\end{array}$} & \multirow[t]{2}{*}{ Accura } & \multirow{2}{*}{\multicolumn{2}{|c|}{$\begin{array}{c}\text { Sensitivity } \\
(95 \% \mathrm{CI})\end{array}$}} & \multirow[t]{2}{*}{$\begin{array}{l}\text { Specificity } \\
(95 \% \mathrm{CI})\end{array}$} & \multirow[t]{2}{*}{$\begin{array}{c}\text { PPV } \\
(95 \% \mathrm{Cl})\end{array}$} & \multirow[t]{2}{*}{$\begin{array}{c}\text { NPV } \\
(95 \% \mathrm{Cl})\end{array}$} \\
\hline & & Positive & Negative & & & & & & \\
\hline \multirow[t]{2}{*}{ S. aureus } & Positive & 82 & 0 & 99. & \multicolumn{2}{|c|}{$98.8 \%$} & \multirow{2}{*}{$\begin{array}{l}100 \% \\
(88.7-100)\end{array}$} & $100 \%$ & $96.8 \%$ \\
\hline & Negative & 1 & 30 & & \multicolumn{2}{|c|}{$(93.5-100)$} & & & $(81.6-99.5)$ \\
\hline
\end{tabular}

PPV, positive predictive value; NPV, negative predictive value; $\mathrm{Cl}$, confidence interval. 
positive samples were from patients who had ESBL-producing K. pneumoniae (blood culture $N=1$; urine culture $N=1$ ) isolated in the subsequent 7 days. The corresponding cultures of the two false-negative samples grew ESBL-producing K. pneumoniae and ESBL-producing Klebsiella oxytoca at quantities of $25,000-50,000 \mathrm{cfu} / \mathrm{mL}$. No other ESBL- or carbapenemase-producing Gram-negative bacteria were detected by either molecular or culture-based testing.

ELITe MGB kit detected S. aureus in 82 of $113(72.6 \%)$ specimens, all of which were confirmed by culture. There was one false-negative sample which gave a $\mathrm{Ct}$ value of 38.2 and a semi-quantitative culture result of $10,000-50,000 \mathrm{cfu} / \mathrm{mL}$. Among the 83 culture-positive $S$. aureus samples, the mecA/C target was found in 23 . The two presumed false-positive samples by PCR may reflect the limitations of phenotypic detection of meticillin resistance [17] or the presence of mixed populations of bacteria. The false-negative sample showed $\Delta \mathrm{Ct}=2.61$, and culture-based diagnostics showed a mixed population of MRSA and meticillin-resistant coagulase-negative staphylococci.

The $\mathrm{Ct}$ values determined by CRE, ESBL and MRSA/SA ELITe MGB kits were compared with the bacterial loads obtained by conventional culture-based approach (Figures 1 and 2, see online supplementary material). The mean Cts obtained by CRE and ESBL ELITe MGB kits were significantly different $(P<0.05)$ between the culture-negative and $>25,000 \mathrm{cfu} / \mathrm{mL}$ groups. With the MRSA/SA ELITe MGB kit, mean Cts for each quantitative culture group were significantly different $(P<0.05)$, except between the $1000-10,000 \mathrm{cfu} / \mathrm{mL}$ and $10,000-50,000 \mathrm{cfu} / \mathrm{mL}$ groups.

\section{Discussion}

Conventional culture-based diagnostics has limitations in tackling the dissemination of multi-drug-resistant pathogens and in optimizing antibiotic therapy in patients with BLRTI in a timely manner. Molecular assays have the potential to perform a role as a more accurate and sensitive decision-making tool, expediting infection control practices and supporting efforts to curtail inappropriate antibiotic use $[12,18,19]$.

This study represents one of the largest performance assessments of molecular assays detecting the main antibiotic resistance genes directly from clinical respiratory samples. High NPV but more variable PPV were found. Several molecular assays of microbiological respiratory diagnostics have focused on rapid pathogen identification but few data on rapid antibiotic resistance have been reported, other than for MRSA [15,20-22]. The CRE and ESBL ELITe MGB kits were particularly suited for the Italian and European epidemiology, as the selection of these samples represents a picture of the most prevalent carbapenemase- and ESBL-producing Enterobacterales [23]. However, the limited number of enzymes tested for would need to be considered if these tests were implemented into clinical practice. The study data indicate that the MRSA/SA ELITe MGB kit, when S. aureus and mec genes are detected at the same relative quantities in the presence of clinical signs of BLRTI, could be of value in guiding the need for anti-MRSA therapy.

The potential role of molecular assays in surveillance, infection control practices and early optimization of antibiotic therapy is well known [12,24,25]. In particular, rapid availability of molecular results can not only facilitate early appropriate antibiotic therapy for patients with multi-drug-resistant bacterial infection, but can also guide earlier de-escalation of antibiotic therapy for patients with negative results. Accuracy rates of the ELITe MGB assays confirm that a molecular approach together with knowledge of local epidemiology susceptibility patterns could be used to expedite optimization of empirical antibiotic therapy and infection control practices in patients with BLRTI, especially when providing positive results for targeted antibiotic resistance genes. However, conventional culture-based antimicrobial susceptibility testing continues to be required to confirm molecular results and to detect other antibiotic resistance mechanisms.

Nucleic acid amplification techniques cannot distinguish between living and dead bacteria [26]. The degree of correlation between bacterial load and $\mathrm{Ct}$ values could be conditional on factors affecting the viability of bacteria at the time of sampling (prior antibiotic treatment, immune-mediated bacterial death [27]), as well as, potentially, the presence of multiple copies of the same gene on mobile genetic elements $[28,29]$. In spite of these limitations, this study showed how Ct analysis may deliver some information about bacterial load.

This study has several limitations, the main one being the lack of clinical data and prospective assessment of direct implications of molecular results on antimicrobial stewardship and clinical outcome. The administration of antibiotics before sampling was not known, and this factor might have hampered the overall evaluation of the molecular false-positive results. Nevertheless, the authors believe that this study shows the potential for the use of CRE, ESBL and MRSA/SA ELITe MGB kits to support infection control and antibiotic stewardship programmes in patients with a high suspicion of multi-drugresistant BLRTI. Any future studies of the effectiveness of this approach will need to consider the feasibility of producing results in a timely manner (testing requires approximately 30 min of laboratory hands-on time and a test run time of approximately $3 \mathrm{~h}$ ), as well as the willingness of clinicians to respond to the results. All of these results would, in turn, have to feed into an assessment of cost effectiveness.

In conclusion, in the smart era of resistance profiling, the ELITe MGB assays showed reasonable accuracy for the detection of carbapenemase- and ESBL-producing Enterobacterales and MRSA in respiratory samples. These tests might be a useful complementary tool for expediting optimization of empirical antibiotic therapy and infection control practices in patients with BLRTI, depending on local prevalence rates of antibiotic resistance. However, further studies are required to confirm these results, to determine robust $\mathrm{Ct}$ cut-off values for colonization vs infection, and to determine their clinical and cost effectiveness in routine clinical practice.

\section{Conflict of interest statement}

None declared.

\section{Funding sources}

None.

\section{Appendix A. Supplementary data}

Supplementary data to this article can be found online at https://doi.org/10.1016/j.jhin.2019.12.025. 


\section{References}

[1] GBD 2013 Mortality and Causes of Death Collaborators. Global, regional, and national age-sex specific all-cause and causespecific mortality for 240 causes of death, 1990-2013: a systematic analysis for the Global Burden of Disease Study 2013. Lancet 2015;385:117-71.

[2] Chastre J, Fagon JY. Ventilator-associated pneumonia. Am J Respir Crit Care Med 2002;165:867-903.

[3] American Thoracic Society, Infectious Diseases Society of America. Guidelines for the management of adults with hospitalacquired, ventilator associated, and healthcare-associated pneumonia. Am J Respir Crit Care Med 2005;171:388-416.

[4] Welte T, Torres A, Nathwani D. Clinical and economic burden of community-acquired pneumonia among adults in Europe. Thorax 2012;67:71-9.

[5] File Jr TM, Marrie TJ. Burden of community-acquired pneumonia in North American adults. Postgrad Med 2010;122:130-41.

[6] Song JH, Thamlikitkul V, Hsueh PR. Clinical and economic burden of community-acquired pneumonia amongst adults in the AsiaPacific region. Int J Antimicrob Agents 2011;38:108-17.

[7] Ewig S, Torres A. Community-acquired pneumonia as an emergency: time for an aggressive intervention to lower mortality. Eur Respir J 2011;38:253-60.

[8] Chalmers JD, Taylor JK, Singanayagam A, Fleming GB, Akram AR, Mandal P. Epidemiology, antibiotic therapy, and clinical outcomes in health care-associated pneumonia: QA UK cohort study. Clin Infect Dis 2011;53:107-13.

[9] Bianco G, Boattini M, lannaccone M, Sidoti F, Cavallo R, Costa C. Detection of antibiotic resistance genes from blood cultures: performance assessment and potential impact on antibiotic therapy management. J Hosp Infect 2019;102:465-9.

[10] Girlich D, Bernabeu S, Fortineau N, Dortet L, Naas T. Evaluation of the CRE and ESBL ELITe MGB ${ }^{\circledR}$ kits for the accurate detection of carbapenemase- or CTX-M-producing bacteria. Diagn Microbiol Infect Dis 2018;92:1-7.

[11] Wang H, Hecht S, Kline D, Leber AL. Staphylococcus aureus and methicillin resistance detection directly from pediatric samples using PCR assays with differential cycle threshold values for corroboration of methicillin resistance. J Microbiol Methods 2019;159:167-73.

[12] Paonessa JR, Shah RD, Pickens Cl, Lizza BD, Donnelly HK, Malczynski M, et al. Rapid detection of methicillin-resistant Staphylococcus aureus in BAL: a pilot randomized controlled trial. Chest 2019;155:999-1007.

[13] Burillo A, Marín M, Cercenado E, Ruiz-Carrascoso G, PérezGranda MJ, Oteo J, et al. Evaluation of the Xpert Carba-R (Cepheid) assay using contrived bronchial specimens from patients with suspicion of ventilator-associated pneumonia for the detection of prevalent carbapenemases. PLoS One 2016;11:e0168473.

[14] Cercenado E, Marín M, Burillo A, Martín-Rabadán P, Rivera M, Bouza E. Rapid detection of Staphylococcus aureus in lower respiratory tract secretions from patients with suspected ventilatorassociated pneumonia: evaluation of the Cepheid Xpert MRSA/SA SSTI assay. J Clin Microbiol 2012;50:4095-7.
[15] Ullberg M, Lüthje P, Mölling P, Strålin K, Özenci V. Broad-range detection of microorganisms directly from bronchoalveolar lavage specimens by PCR/electrospray ionization-mass spectrometry. PLoS One 2017;12:e0170033.

[16] EUCAST 2020. Available at: www.eucast.org/fileadmin/src/ media/PDFs/EUCAST_files/Breakpoint_tables/v_10.0_ Breakpoint_Tables.pdf [last accessed February 2020].

[17] Kriegeskorte A, Idelevich EA, Schlattmann A, Layer F, Strommenger B, Denis O, et al. Comparison of different phenotypic approaches to screen and detect mecC-harboring methicillin-resistant Staphylococcus aureus. J Clin Microbiol 2017;56: e00826-17.

[18] Torres A, Lee N, Cilloniz C, Vila J, Van der Eerden M. Laboratory diagnosis of pneumonia in the molecular age. Eur Respir $J$ 2016;48:1764-78.

[19] Douglas IS. New diagnostic methods for pneumonia in the ICU. Curr Opin Infect Dis 2016;29:197-204.

[20] Jamal W, Al Roomi E, AbdulAziz LR, Rotimi VO. Evaluation of Curetis Unyvero, a multiplex PCR-based testing system, for rapid detection of bacteria and antibiotic resistance and impact of the assay on management of severe nosocomial pneumonia. J Clin Microbiol 2014;52:2487-92.

[21] Pulido MR, Moreno-Martínez P, González-Galán V, Fernández Cuenca F, Pascual Á, Garnacho-Montero J, et al. Application of BioFire FilmArray blood culture identification panel for rapid identification of the causative agents of ventilator-associated pneumonia. Clin Microbiol Infect 2018;24:1213.e1-4.

[22] Sansot M, Fradin E, Chenouard R, Kempf M, Kouatchet A, Lasocki S, et al. Performance of the extended use of the FilmArray (®) BCID panel kit for bronchoalveolar lavage analysis. Mol Biol Rep 2019;46:2685-92.

[23] Bevan ER, Jones AM, Hawkey PM. Global epidemiology of CTX-M $\beta$-lactamases: temporal and geographical shifts in genotype. J Antimicrob Chemother 2017;72:2145-55.

[24] Saliba R, Aho-Glélé LS, Karam-Sarkis D, Zahar JR. Evaluation of polymerase chain reaction assays for direct screening of carbapenemase-producing Enterobacteriaceae from rectal swabs: a diagnostic meta-analysis. J Hosp Infect 2020;104:381-9.

[25] van Belkum A, Rochas O. Laboratory-based and point-of-care testing for MSSA/MRSA detection in the age of whole genome sequencing. Front Microbiol 2018;9:1437.

[26] Schulte B, Eickmeyer H, Heininger A, Juretzek S, Karrasch M, Denis 0 , et al. Detection of pneumonia associated pathogens using a prototype multiplexed pneumonia test in hospitalized patients with severe pneumonia. PLoS One 2014;9:e110566.

[27] Guimaraes AO, Gutierrez J, Maskarinec SA, Cao Y, Hong K, Ruffin $F$, et al. Prognostic power of pathogen cell-free DNA in Staphylococcus aureus bacteremia. Open Forum Infect Dis 2019;6:ofz126.

[28] D’Andrea MM, Arena F, Pallecchi L, Rossolini GM. CTX-M-type $\beta$ lactamases: a successful story of antibiotic resistance. Int J Med Microbiol 2013;303:305-17.

[29] Yigit H, Queenan AM, Anderson GJ, Domenech-Sanchez A, Biddle JW, Steward CD, et al. Novel carbapenem-hydrolyzing beta-lactamase, KPC-1, from a carbapenem-resistant strain of Klebsiella pneumoniae. Antimicrob Agents Chemother 2001;45:1151-61. 